\title{
The association of CMTM6 expression with prognosis and PD-L1 expression in triple-negative breast cancer
}

\author{
Yiping Tian ${ }^{1,2 \#}$, Xiaohui Sun ${ }^{3 \#}$, Guoping Cheng ${ }^{1,2}$, Enming Ji1 ${ }^{1,2}$, Shifeng Yang ${ }^{1,2}$, Jianguo Feng ${ }^{2,4}$, Linfeng Zheng $^{1,2}$ \\ ${ }^{1}$ Department of Pathology, Cancer Hospital of the University of Chinese Academy of Sciences (Zhejiang Cancer Hospital), Hangzhou, China; \\ ${ }^{2}$ Institute of Cancer and Basic Medicine (ICBM), Chinese Academy of Sciences, Hangzhou, China; ${ }^{3}$ Department of Epidemiology and Biostatistics, \\ School of Public Health, Zhejiang Chinese Medical University, Hangzhou, China; ${ }^{4}$ Department of Experimental Research Center, Cancer Hospital \\ of the University of Chinese Academy of Sciences (Zhejiang Cancer Hospital), Hangzhou, China \\ Contributions: (I) Conception and design: L Zheng, Y Tian; (II) Administrative support: L Zheng; (III) Provision of study materials or patients: L \\ Zheng, Y Tian, E Ji, S Yang; (IV) Collection and assembly of data: G Cheng, J Feng; (V) Data analysis and interpretation: X Sun, Y Tian; (VI) \\ Manuscript writing: All authors; (VII) Final approval of manuscript: All authors. \\ \#These authors contributed equally to this work. \\ Correspondence to: Linfeng Zheng. Department of Pathology, Cancer Hospital of the University of Chinese Academy of Sciences (Zhejiang Cancer \\ Hospital), Hangzhou, China; Institute of Basic Medicine and Cancer (IBMC), Chinese Academy of Sciences, No. 1 East Banshan Road, Gongshu \\ District, Hangzhou, China. Email: zhenglf@zjcc.org.cn.
}

Background: Immune checkpoint inhibitors play a vital role in triple-negative breast cancer (TNBC) immunotherapy. A recent study showed that chemokine-like factor (CKLF)-like MARVEL transmembrane domain containing 6 (CMTM6) has a crucial role in programmed death-ligand 1 (PD-L1) stability. The aim of this study was to investigate the relationship between CMTM6 and PD-L1 in TNBC and the association with clinical characteristics.

Methods: A total of 143 patients, including 75 with human epidermal growth factor receptor 2 (HER2)driven breast cancer and 68 with TNBC, were included in this study. In 83 paired primary breast cancers (PBCs) and metastatic breast cancers (MBC) comprising 45 HER2-driven breast cancers and 38 TNBC, CMTM6 and PD-L1 were detected based on immunohistochemistry (IHC) with FFPE tissues. Another 60 PBCs comprising 30 HER2-driven breast cancers and 30 TNBC in order to detect CMTM6 and PDL1 mRNA expressions based on real-time polymerase chain reaction (RT-PCR) using frozen tissues. Furthermore, 153 patients comprising 30 TNBC and 123 HER2-driven breast cancer based on The Cancer Genome Atlas (TCGA) database were used to confirm the difference mRNA expression.

Results: The expression of CMTM6 in patients with TNBC was significantly higher than in those with HER2-driven PBC (IHC, P=0.036, mRNA, P=0.036, TCGA dataset, P=0.039). CMTM6 was correlated with PD-L1 based on IHC in triple-negative MBC $(\mathrm{P}=0.004)$; the same result was found based on mRNA data in triple- negative $\mathrm{PBC}(\mathrm{P}=0.021)$. Moreover, a high expression of CMTM6 in TNBC was associated with poor progression-free survival (PFS) (P=0.030, 95\% CI: 1.08-4.57, HR =2.22). After multiple Cox regression analysis, CMTM6 in TNBC emerged as an independent risk factor for PFS ( $\mathrm{P}=0.027,95 \%$ CI: 1.11-5.20, HR =2.40). The expression of PD-L1 was negatively correlated with lymph node metastasis $(\mathrm{P}=0.026)$ and was not associated with PFS.

Conclusions: The expression of CMTM6 was higher in TNBC than in HER2-driven breast cancer. In TNBC, CMTM6 was correlated with PD-L1 expression, and potentially could be used as an independent risk factor for predicting PFS.

Keywords: CMTM6; PD-L1; triple-negative breast cancer (TNBC); immunohistochemistry (IHC); polymerase chain reaction (PCR); prognosis

Submitted Oct 16, 2020. Accepted for publication Jan 14, 2021.

doi: 10.21037/atm-20-7616

View this article at: http://dx.doi.org/10.21037/atm-20-7616 


\section{Introduction}

Breast cancer is the most common malignancy in women worldwide and its clinical and molecular heterogeneity is well-documented. Endocrine receptors for estrogen (ER) and progesterone (PR), and the aberrant expression of human epidermal growth factor receptor-2 (HER2) are the specific biomarkers for breast cancer most commonly used in clinical practice. Triple-negative breast cancer (TNBC), which lacks expression of ER, PR, and HER2, is found in $10-20 \%$ of all breast cancers. TNBC is an aggressive type of breast cancer that usually displays a higher grade and poorer outcome than other breast cancer subtypes $(1,2)$. Therefore, effective therapeutic strategies for TNBC are urgently needed.

Programmed death-ligand 1 (PD-L1) is a ligand of the programmed cell death-1 (PD-1) receptor and can be expressed by the tumor cell surface as well as by tumor infiltration lymphocytes (TILs). Binding of PD-L1 to PD-1 can downregulate anti-tumor $\mathrm{T}$ cell responses which lead to tumor immune escape (3). To date, immune checkpoint inhibitors that block the interaction between PD-1 and PDL1 have been used to treat many types of metastatic cancers (3-5). In primary breast cancer (PBC), the expression of PD-L1 is heterogeneous and associated with higher histological grades and more aggressive molecular subtypes [triple-negative (TN), basal, and HER2-driven] $(6,7)$. However, the regulation mechanism of PD-L1 remains elusive.

Recently, chemokine-like factor (CKLF)-like Marvel Transmembrane Domain-containing 6 (CMTM6) has been identified as a key regulator of the $\mathrm{PD}-\mathrm{L} 1$ protein, which is also thought to be involved in modulating tumor immunity $(8,9)$. Dysfunction of CMTM6 impairs the expression of PD-L1 in many human tumor cell types, such as lung cancer, thyroid cancer, and melanoma (8). The depletion of CMTM6, via downregulated PD-L1, can reduce the suppression of tumor-specific $\mathrm{T}$ cell activity in vitro and in vivo assays (10). Previous studies have suggested the potential value of a therapeutic target that elicits an immune response and avoids the escape of immune surveillance. However, the role of CMTM6 in breast cancer remains unclear.

In the present study, we used immunohistochemistry (IHC) and real-time polymerase chain reaction (RT-PCR) to analyze the correlation between CMTM6 and PDL1 in patients with TNBC. Furthermore, we assessed the association of CMTM6 or PD-L1 expression with patients' prognosis. This study gives a hint that the expression of CMTM6 may become a potential biomarker of immunotherapies through supplement to PD-L1 expression and could predict the patients' outcome. We present the following article in accordance with the REMARK reporting checklist (available at http://dx.doi.org/10.21037/ atm-20-7616).

\section{Methods}

\section{Patients}

A total of 143 patients with breast cancer who underwent surgical resection in the Cancer Hospital of the University of Chinese Academy of Sciences in China between January 2008 and December 2015 were enrolled in the study. Of these, 83 patients who had paired primary breast cancer tissues and matched metastatic cancer tissues were selected and comprised 45 HER2-driven breast cancer and 38 TNBC patients. All of these patients were without neoadjuvant chemotherapy at the first diagnosis and had sufficient archival tissue in formalin-fixed, paraffinembedded (FFPE) blocks to perform IHC analyses. The remaining 60 individuals comprised 30 HER2-driven and 30 TNBC PBC patients with sufficient frozen tissue to perform mRNA expression detection (Figure S1).

The eighth edition of the tumor node metastasis (TNM) classification of the American Joint Commission on Cancer (AJCC) was used as a reference for pathological features and clinical stage. Progression-free survival (PFS) was defined as the time from the date of the initial treatment until the date of diagnosis of the initial recurrence or death from any cause. The PFS data were locked on August 30, 2020.

This study was approved by the Medical Ethics Committee of Zhejiang Cancer Hospital (IRB-2020275). The study was conducted in accordance with the Declaration of Helsinki (as revised in 2013). Because of the retrospective nature of the research, the requirement for informed consent was waived.

\section{IHC staining of CMTM6 and PD-L1}

FFPE tissue specimens were collected from 83 patients with paired $\mathrm{PBC}$ and metastatic cancer tissues, and an IHC assay was performed to detect the expression of CMTM6 and PD-L1. FFPE tissue blocks were cut into 4- $\mu \mathrm{m}$-thick sections and attached to a positively charged glass slide. Immunohistochemical staining of CMTM6 was carried out 
with a Leica BOND-III automatic IHC staining device. The samples were incubated with an antibody against CMTM6 for $15 \mathrm{~min}$ at room temperature (dilution 1:200, recombinant monoclonal antibody, Abcam, EPR2301545 , US). The signal was subsequently detected with a Leica Bond Polymer Refine Detection Kit (Leica Biosystems, Buffalo Grove, US). Post-primary antibody incubation and polymer incubation were set at $8 \mathrm{~min}$ for CMTM6 and followed by immersion in diaminobenzidine (DAB) for signal visualization for $10 \mathrm{~min}$ at room temperature. CMTM6 expression was defined as any intensity in the cytomembrane on the tumor cells (Figure S2A,B).

For PD-L1 staining, the VENTANA PD-L1 (SP142) rabbit monoclonal primary antibody (Ventana Medical Systems Inc., Tucson, AZ, USA) was optimized for use as a fully automated IHC assay on the BenchMark ULTRA (Ventana Medical Systems Inc., Tucson, AZ, USA) staining platform using the OptiView DAB IHC Detection Kit and OptiView Amplification Kit (Ventana Medical Systems Inc., Tucson, AZ, USA) as previously described (11). PD-L1 expression was identified as any intensity in the cytomembrane on the tumor-infiltrating immune cells (Figure S2C,D).

\section{RNA extraction and quantitative RT-PCR}

Total RNA was isolated from 60 frozen cancer tissues using TRIzol (Invitrogen, USA), following the manufacturer's protocol. The concentration of RNAs was measured through a microvolume spectrophotometer. The 500 nanograms of RNA was reverse transcribed using a PrimeScript $^{\mathrm{TM}}$ RT Reagent Kit (Takara, Dalian, China). Quantitative PCR (qPCR) was carried out as previously described (12). The primer sequences were: CMTM6-F: 5 '-GCAACAATATCAGCAACTTCGT-3' and CMTM6-R: 5'-TTGGTCCTTAGGTGTGGTATCA-3'; PD-L1-F: 5'-CACCACCACCAATTCCAAGAG-3'; PD-L1-R: 5'- AGGATGTGCCAGAGGTAGTTC-3'; $\beta$-actin-F: 5 '-TGGCACCCAGCACAATGAA-3', $\beta$-actin-R: 5 '-CTAAGTCATAGTCCGCCTAGAAG CA-3'.

\section{Western blotting}

Cells were collected and prepared as described earlier (13). Proteins were separated by $10 \%$ SDS-PAGE and transferred to polyvinylidene fluoride membrane (Millipore, Bedford, MA, USA). Membranes were blocked in 5\% non- fat milk in TBS-Tween 20 for 2 hours prior to overnight incubation with primary antibodies at $4{ }^{\circ} \mathrm{C}$, and were then incubated for 2 hours at room temperature with the secondary antibodies (Servicebio, Wuhan, China). Finally, the protein level was detected using an enhanced chemiluminescence (ECL) reagent (CwBio, Beijing, China). Glyceraldehyde 3-phosphate dehydrogenase (GAPDH) was used for normalization. Primary antibodies were directed against CMTM6 (Abcam, USA, 1:1,000), PD-L1 (Proteintech, USA, 1:1,000), and GAPDH (Santa Cruz, CA, USA, 1: 5,000).

\section{Web-based mRNA profiling}

The RNA-sequencing data and clinical records of 30 HER2-driven PBC and 123 triple-negative PBC patients were downloaded from The Cancer Genome Atlas (TCGA) dataset (see URLs http://cancergenome.nih.gov/). The present study conforms to the publication guidelines.

\section{Statistical analysis}

Statistical analysis was performed using SPSS statistics version 22.0 (IBM Corp., Armonk, NY, USA). The continuous variables were described as means and standard deviations (SD), and the categorical variables were described as number (percentage). Any skewed distribution data were expressed with median and interquartile ranges. The Mann-Whitney test was adopted to evaluate the nonnormal distribution dataset. Spearman's correlation analysis was used to describe the correlation between quantitative variables with skewed distribution data, and the Pearson Chi-square and McNemar's tests were used to compare categorical variables. Kaplan-Meier (K-M) analysis was used, and between-group differences were evaluated by the log-rank test. The multivariable Cox proportional hazard model was applied to calculate the adjusted hazard ratio (HR). A two-sided $\mathrm{P}$ value $<0.05$ was considered statistically significant.

\section{Results}

\section{Patient characteristics}

A total of 143 patients with breast cancer, including 75 (52.4\%) HER2-driven PBCs and 68 (47.6\%) triple-negative PBCs were included in the study. The clinical features are shown in Table 1. All patients were female, with a mean 
Table 1 General clinical characteristics

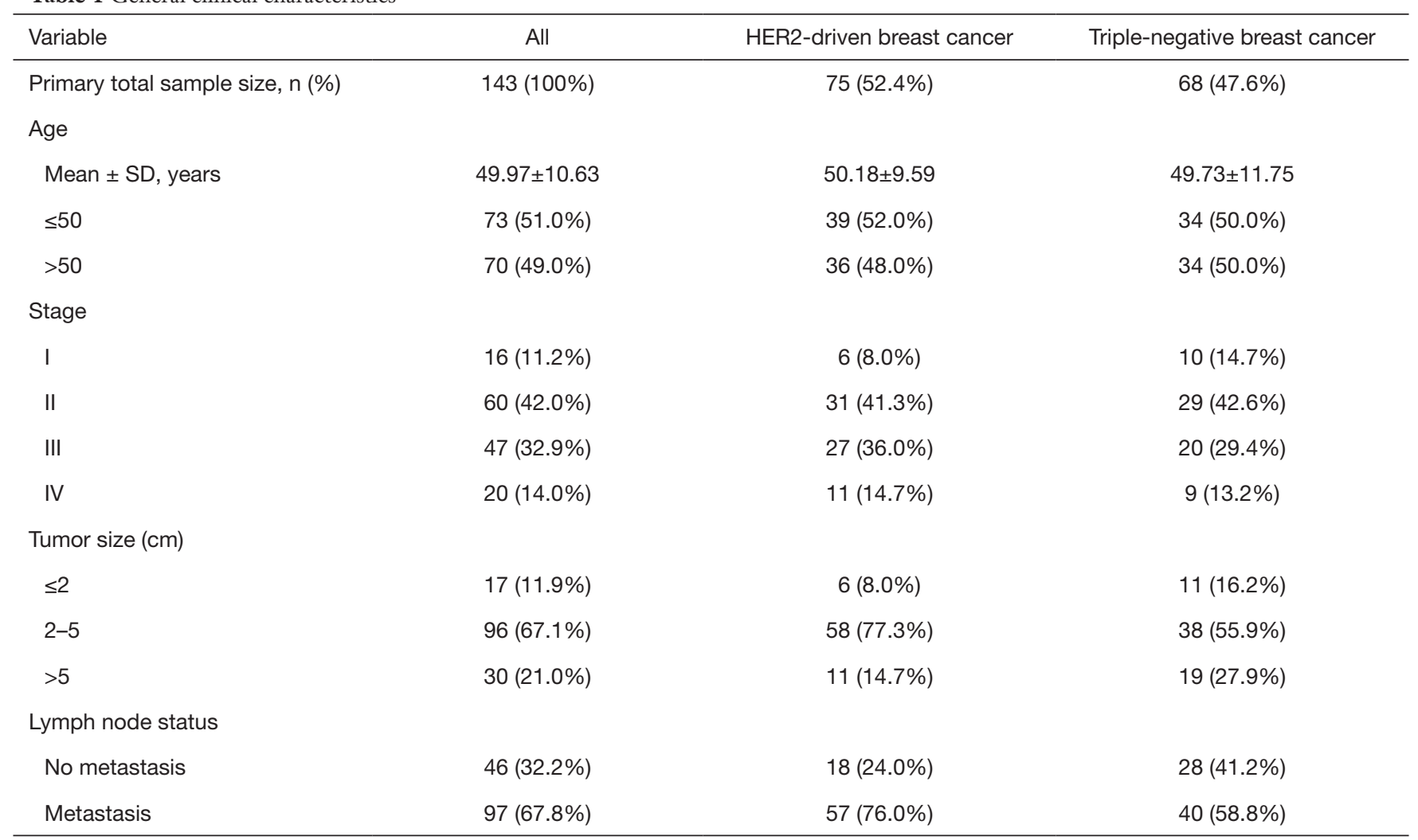

age of $49.97 \pm 10.63$ years. Of these, $16(11.2 \%)$ had stage I, $60(42.0 \%)$ had stage II, $47(32.9 \%)$ had stage III, and 20 (14.0\%) had stage IV diagnoses of breast cancer. A total of $97(67.8 \%)$ patients showed lymph node metastases. There were $17(11.9 \%)$ patients with a tumor size smaller than $2 \mathrm{~cm}, 96(67.1 \%)$ patients with a tumor size of $2-5 \mathrm{~cm}$, and $30(21.0 \%)$ patients with a tumor size larger than $5 \mathrm{~cm}$. Of the total number of 143 patients, 83 had matched $\mathrm{PBC}$ and metastatic breast cancer (MBC). There was a 97.6\% (81/83) concordance between ER, PR, and HER2 expression between the matched PBC and MBC patients. The median PFS of these 83 patients was 48 months (range, 2976 months).

\section{CMTM6 and PD-L1 expression in HER2-driven breast cancer and TNBC}

IHC was performed to test the expression of CMTM6 and PD-L1 in the 83 paired breast cancers. The typical CMTM6 and PD-L1 staining images from breast cancer samples are shown in Figure S2. Western blot was used to confirm the expression detected by IHC (Figure S3).
In PBC, 56 (67.5\%) cases were negative for CMTM6, while 27 (32.5\%) showed CMTM6-positive staining. The positive expression rate of CMTM6 in patients with triplenegative $\mathrm{PBC}$ was significantly higher than in those with HER2-driven PBC (63.0\% vs. 37.0\%, $\mathrm{P}=0.036$, Table 2). A similar result was found in the $\mathrm{MBC}$ patients $(61.1 \%$ vs. $38.9 \%, \mathrm{P}=0.048$, Table 2). Moreover, the mRNA expression level of CMTM6 showed the same result (the median CMTM6 mRNA expression was 0.037 in triple-negative $\mathrm{PBC}$ and 0.026 in HER2-driven PBC, $\mathrm{P}=0.036$, Table S1, Figure S4A).

For the PD-L1 expression, 50 (60.2\%) showed negative staining, while 33 (39.8\%) showed positive staining. However, no significant difference in PD-L1 expression was found between HER2-driven breast cancer and TNBC, either in $\mathrm{PBC}$ or in MBC samples (Table 2). Moreover, a significant difference was found in the PD-L1 mRNA expression level between triple-negative PBC and HER2driven PBC (the median expression level was 0.00026 and 0.00012, respectively, $\mathrm{P}=0.040$, Table S1, Figure S4B).

To further verify our findings, we performed CMTM6 and PD-L1 mRNA expression analyses in HER2-driven 
Table 2 CMTM6 and PD-L1 protein expression in HER2-driven breast cancer and triple-negative breast cancer

\begin{tabular}{|c|c|c|c|c|c|c|}
\hline Protein expression status & \multicolumn{3}{|c|}{ Primary } & \multicolumn{3}{|c|}{ Metastasis } \\
\hline CMTM6 negative & $35(62.5)$ & $21(37.5)$ & \multirow{2}{*}{0.036} & $29(61.7)$ & $18(38.3)$ & \multirow{2}{*}{0.048} \\
\hline CMTM6 positive & $10(37.0)$ & $17(63.0)$ & & $14(38.9)$ & $22(61.1)$ & \\
\hline PD-L1 negative & $28(56.0)$ & $22(44.0)$ & 0.822 & $31(50.0)$ & $31(50.0)$ & 0.621 \\
\hline
\end{tabular}

Pearson Chi-square test.

Table 3 CMTM6 and PD-L1 protein expression in matched PBC and MBC

\begin{tabular}{|c|c|c|c|c|c|c|}
\hline $\begin{array}{l}\text { Matched MBC protein } \\
\text { expression status }\end{array}$ & \multicolumn{3}{|c|}{ CMTM6 expression in primary cancer } & \multicolumn{3}{|c|}{ PD-L1 expression in primary cancer } \\
\hline \multicolumn{7}{|l|}{ All metastasis samples } \\
\hline Negative & $39(83.0)$ & $8(17.0)$ & & $43(69.4)$ & $19(30.6)$ & \\
\hline Positive & $17(47.2)$ & $19(52.8)$ & 0.108 & 7 (33.3) & $14(66.7)$ & 0.031 \\
\hline Negative & $26(86.7)$ & $4(13.3)$ & & $22(66.7)$ & $11(33.3)$ & \\
\hline Positive & $9(60.0)$ & $6(40.0)$ & 0.267 & $6(50.0)$ & $6(50.0)$ & 0.332 \\
\hline \multicolumn{7}{|c|}{ Metastasis triple-negative samples } \\
\hline Negative & $13(76.5)$ & $4(23.5)$ & & $21(72.4)$ & $8(27.6)$ & \\
\hline
\end{tabular}

McNemar's test. PBC, primary breast cancer; MBC, metastatic breast cancer.

PBC and triple-negative PBC using the TCGA dataset. Although the expression level of PD-L1 between HER-2 driven $\mathrm{PBC}$ and triple-negative $\mathrm{PBC}$ did not differ $(\mathrm{P}=0.283$, Table S2), a higher expression level of CMTM6 was observed in triple-negative $\mathrm{PBC}(\mathrm{P}=0.039$, Table S2).

\section{CMTM6 and PD-L1 expression in the matched PBC and MBC samples}

As shown in Table 3, we evaluated whether there was a difference in the expression levels of CMTM6 and PD$\mathrm{L} 1$ in the matched $\mathrm{PBC}$ and MBC samples. Across the 83 matched samples, patients with a $\mathrm{PD}-\mathrm{L} 1$ positive expression in the MBC group were more likely to have a PD-L1 positive expression in the matched $\mathrm{PBC}$ group $(\mathrm{P}=0.031)$. A similar result was found in the triple-negative samples $(\mathrm{P}=0.039)$, while no significant difference was found for the HER2-driven samples. There was no correlation between the expression of CMTM6 in the matched breast cancer samples in this study.

\section{Correlation of CMTM6 expression and PD-L1 expression in breast cancers}

The correlation between the expression of CMTM6 and PD-L1 was further analyzed. In MBC, we found that CMTM6 protein expression was positively correlated with PD-L1 protein expression. McNemar's test showed that the expression of CMTM6 and PD-L1 were significantly correlated across the entire MBC sample $(\mathrm{P}=0.012$, Table 4), but especially in the triple-negative MBC sample $(\mathrm{P}=0.004$, Table 4). However, no significant correlation was observed in the individual PBC or HER2-driven MBC groups (Table 4).

Next, we performed correlation analyses between CMTM6 and PD-L1 expression in the mRNA data using 
Table 4 The associated protein expression between CMTM6 and PD-L1 in breast cancers

\begin{tabular}{|c|c|c|c|c|c|c|}
\hline CMTM6 expression status & \multicolumn{3}{|c|}{ PD-L1 expression in primary cancer } & \multicolumn{3}{|c|}{ PD-L1 expression in metastasis } \\
\hline \multicolumn{7}{|l|}{ CMTM6 in all samples } \\
\hline Negative & $38(67.9)$ & $18(32.1)$ & & $39(83.0)$ & $8(17.0)$ & \\
\hline Positive & $12(44.4)$ & $15(55.6)$ & 0.361 & $23(63.9)$ & $13(36.1)$ & 0.012 \\
\hline Negative & $23(65.7)$ & $12(34.3)$ & & $24(80.0)$ & $6(20.0)$ & \\
\hline Positive & $5(50.0)$ & $5(50.0)$ & 0.143 & $9(60.0)$ & $6(40.0)$ & 0.607 \\
\hline \multicolumn{7}{|c|}{ CMTM6 in triple-negative samples } \\
\hline Negative & $15(71.4)$ & $6(28.6)$ & & $15(88.2)$ & $2(11.8)$ & \\
\hline
\end{tabular}

McNemar's test.

Spearman's correlation coefficient analysis. The results showed that the mRNA expression level of CMTM6 was positively correlated with the mRNA expression level of PD-L1 in total breast cancers ( $\mathrm{r}_{\text {Spearman }}=0.419$ and $\mathrm{P}=0.001$, Figure $1 A$ ), in HER2-driven $\mathrm{PBC}\left(\mathrm{r}_{\text {Spearman }}=0.373\right.$ and $\mathrm{P}=0.042$, Figure $1 B)$, and in TNBC $\left(\mathrm{r}_{\text {Spearman }}=0.421\right.$ and $\mathrm{P}=0.021$, Figure $1 C$ ).

\section{Clinicopathological characteristics by CMTM6 and PD-L1 expression}

CMTM6 expression was not significantly associated with clinicopathological characteristics (Table 5) but was significantly associated with PFS. In the total sample, those with CMTM6 expression were at higher risk for disease progression compared with those without CMTM6 expression, especially in TNBC (HR $=1.83,95 \%$ CI: 1.13 2.96, $\mathrm{P}=0.014$ for the total sample; and $\mathrm{HR}=2.22$; $95 \% \mathrm{CI}$ : 1.08-4.57, $\mathrm{P}=0.030$ for TNBC, Table 6). Median PFS times for those with CMTM6 expression in the total sample and triple-negative cases were 46.8 (IQR, 38.3-50.0) and 47.0 (IQR, 39.5-52.7) months, respectively, while the median for those without CMTM6 expression was over 51 months. The Kaplan-Meier survival analysis demonstrated similar results (Figure 2A,B,C). After adjusting for age and TNM stage, significant associations were found in CMTM6 ( $\mathrm{HR}=2.21,95 \%$ CI: $1.32-3.71, \mathrm{P}=0.003)$, especially in the TNBC patients (HR $=2.40,95 \%$ CI: $1.11-5.20, \mathrm{P}=0.027$, Table 6).

In the case of PD-L1, the absence of PD-L1 expression was correlated with lymph node metastasis $(\mathrm{P}=0.026$, Table 5). No other clinicopathologic characteristics were associated with PD-L1 expression. Moreover, our data showed that the expression of PD-L1 was not significantly associated with disease progression (Figure 2D,E,F, Table 6).

\section{Discussion}

Our data suggested that the expression of CMTM6 was higher in TNBC than in HER2-driven breast cancer and that CMTM6 was also an independent risk factor of PFS, especially in TNBC. The expression level of CMTM6 was positively correlated with the expression of PD-L1. Moreover, the expression of PD-L1 in triple-negative metastatic breast cancer was positively correlated with that in primary breast cancer. And the expression of PD-L1 was negative with metastases in lymph nodes in PBC.

TNBC has a more aggressive biological behavior than other types of breast cancer. Patients with TNBC do not benefit from hormonal or trastuzumab-based therapy because of the loss of the target receptors HER2, ER, and PGR $(2,14)$; chemotherapy and surgery appear to be the only available treatment modalities (15). Recent studies have shown that TNBC has a higher mutational burden than other subtypes and presents with tumorinfiltrating lymphocytes (TILs). Moreover, patients with TNBCs combined low-TILs and high PD-L1 expression showed unfavorable outcome, which may be benefit from immune therapy $(1,7,16-18)$. According to these results, many clinical trials are currently evaluating the role of 

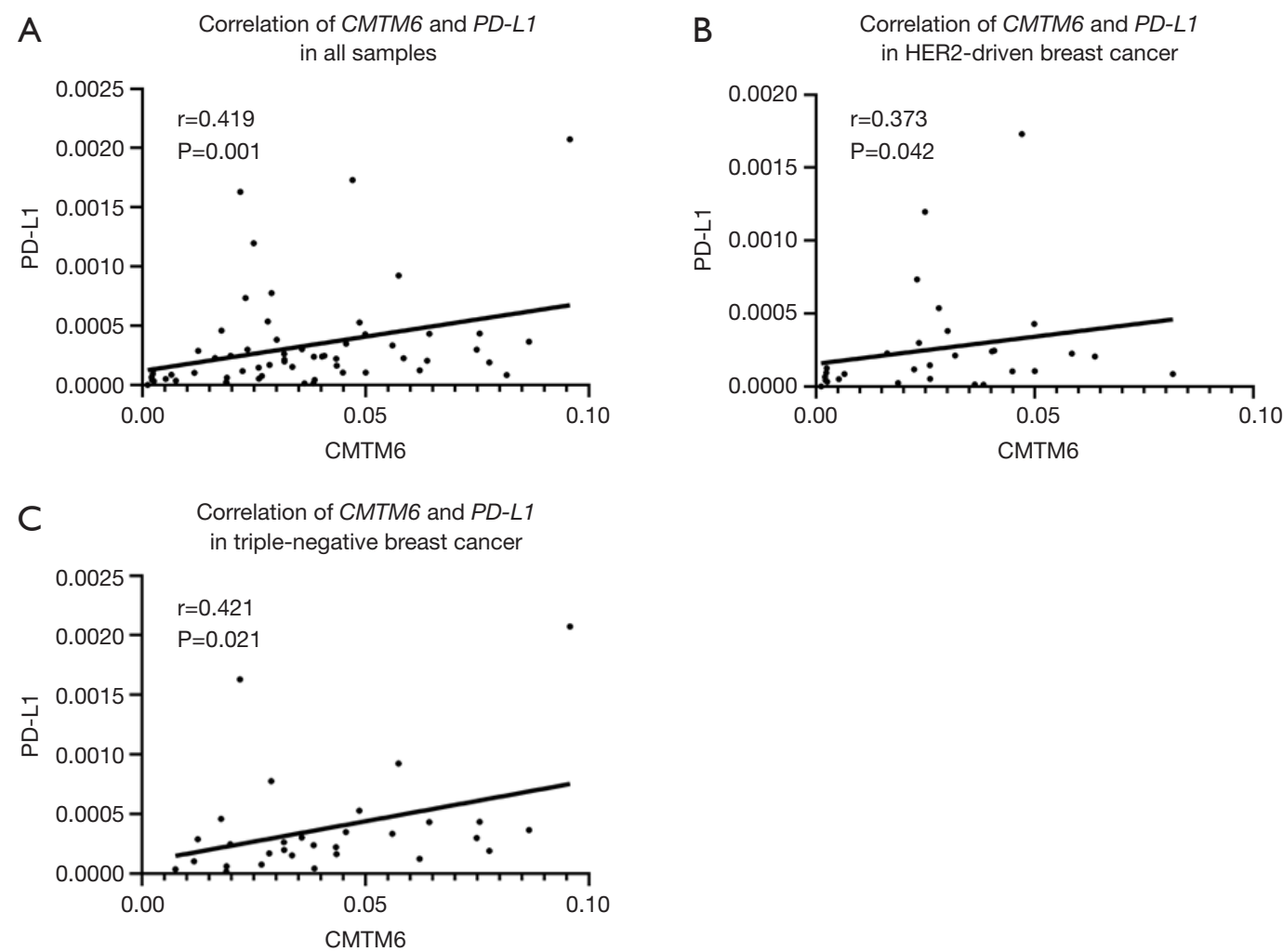

Figure 1 The correlation of CMTM6 and PD-L1 mRNA expression in all 60 samples (A), in HER2-driven breast cancer (B), and in triplenegative breast cancer $(\mathrm{C})$. $\mathrm{P}$ values for correlation analysis are determined with the Spearman correlation coefficient.

Table 5 Clinical features by CMTM6 and PD-L1 protein expression

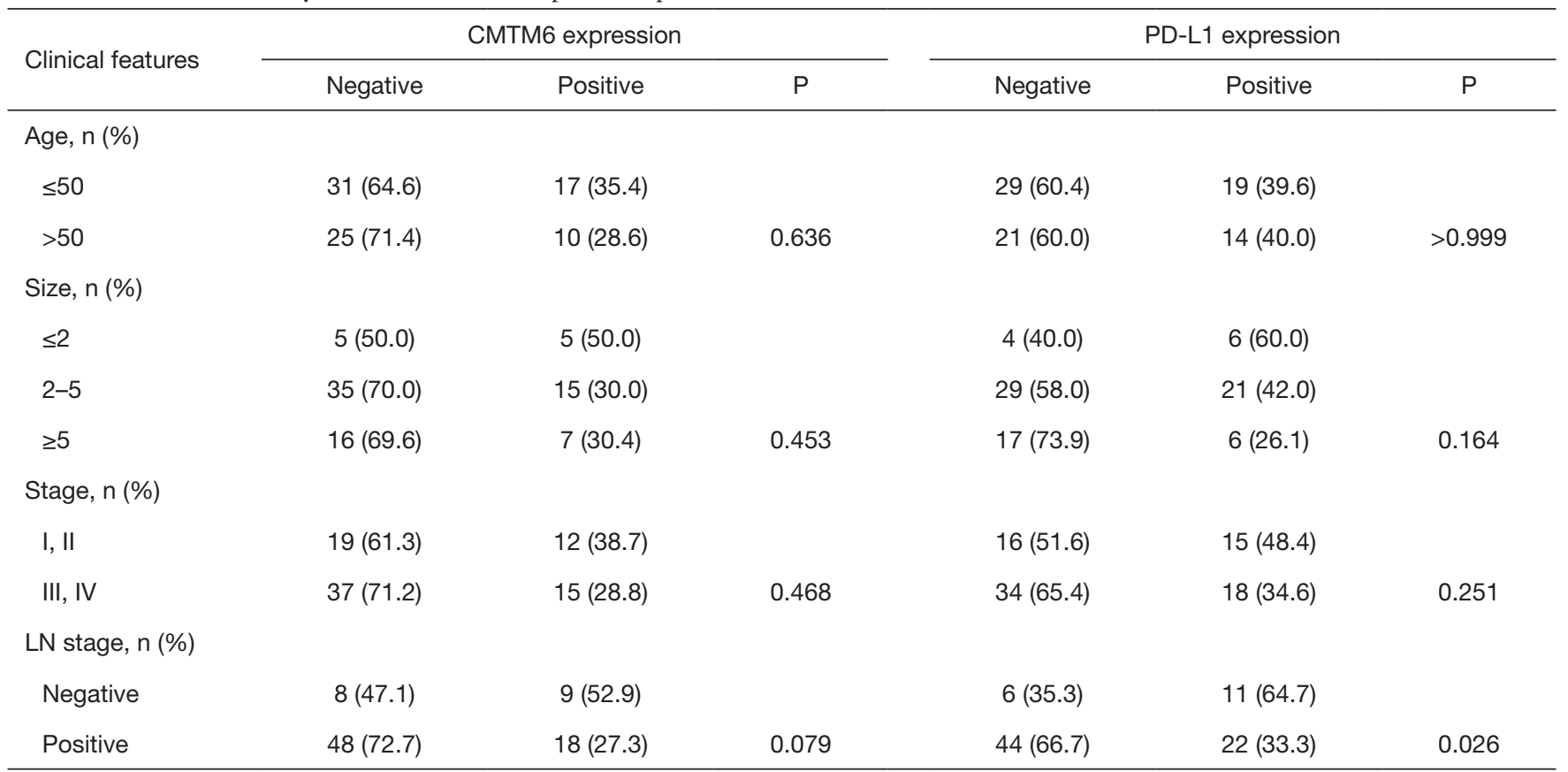

Pearson Chi-square test. 
Table 6 Survival analysis of gene expressions in PBC

\begin{tabular}{llll}
\hline Variable & $\mathrm{HRa}(95 \% \mathrm{Cl})$ & $\mathrm{Pa}$ & $\mathrm{HRb}(95 \% \mathrm{Cl})$ \\
\hline All samples & $1.83(1.13-2.96)$ & 0.014 & $2.21(1.32-3.71)$ \\
CMTM6 & $1.06(0.68-1.65)$ & 0.795 & \\
PD-L1 & $1.83(0.89-3.76)$ & 0.102 & \\
HER2-driven & $0.81(0.43-1.49)$ & 0.490 & \\
CMTM6 & & & \\
PD-L1 & $2.22(1.08-4.57)$ & 0.030 & $2.40(1.11-5.20)$ \\
Triple-negative & $1.36(0.70-2.61)$ & 0.364 & \\
CMTM6 & & & \\
PD-L1 & & & \\
\hline
\end{tabular}

$\mathrm{Pa}$ value for PFS was determined with Cox proportional hazards regression. $\mathrm{Pb}$ value was measured by multivariate analyses of PFS (Cox proportional hazards regression model) after adjustment for age and TNM stage. HRa, hazard ratio (HR) was measured by Cox proportional hazard model; HRb, the adjusted hazard ratio (HR) was measured by multivariate Cox proportional hazard model after adjustment for age and TNM stage. PBC, primary breast cancer; PFS, progression-free survival.

checkpoint inhibitors, PD-1, and/or PD-L1 in TNBC with encouraging results $(19,20)$.

Although anticancer therapies based on immune checkpoint inhibitors targeting the PD-1/PD-L1 pathway have emerged as a critical treatment option, it seems that only a small subset of patients can benefit from these $(20,21)$. To solve this challenge, some studies have focused on investigating the regulation of PD-L1 expression identifying a type- 3 transmembrane protein, CMTM6, as a regulator of PD-L1 expression $(8,10)$. In our study, the expression of CMTM6 was higher in TNBC than in HER2-driven breast cancer, according to the TCGA dataset. The expression of CMTM6 was positively correlated with PD-L1 in PBC and MBC in mRNA expression and IHC data, respectively. Koh et al. and Gao et al. reported similar results in non-small cell lung cancer $(9,22)$. Moreover, our data revealed no significantly different expression of CMTM6 between paired triplenegative $\mathrm{PBC}$ and $\mathrm{MBC}$. Considering the transient PD-L1 expression in tumor cells which tends to disappear rapidly, the intratumoral heterogeneity of PD-L1 expression is frequently observed (23). Thus, the expression of CMTM6 may be a new potential biomarker of immunotherapies in TNBC.

In our study, TNBC patients with a high expression of CMTM6 had a poorer PFS. CMTM6 could therefore serve as an independent risk factor to predict patient outcome. At the mRNA expression level, Mamessier et al. found that a CMTM6-high group had a shorter overall survival than a CMTM6-low group in pancreatic adenocarcinomas. They also found that CMTM6-high and PD-L1-high groups were associated with better metastasisfree survival in triple-negative PBC and that the CMTM6 expression enhanced the prognostic value of PD-L1 expression (24). Furthermore, some studies reported that individuals diagnosed with non-small cell lung cancer and hepatocellular carcinoma who showed a high expression of CMTM6 had better overall survival $(22,25)$. On the other hand, some studies have provided evidence that a high expression of CMTM6 is associated with poor prognosis in gliomas and gastric cancer $(26,27)$. Tumor heterogeneity and ethnic differences may be responsible for these conflicting results, and a larger cohort of a specific cancer type based on multicenter studies is recommended.

This study has several limitations. Firstly, this is a retrospective study with a relatively small sample size. A larger cohort of TNBC patients and multicenter studies are recommended for future studies. Secondly, some cases were from biopsy samples that showed few tumor-infiltrating immune cells, which may explain the lack of significant correlation between the CMTM6 and PD-L1 protein expression in primary TNBC. Moreover, it was not possible to evaluate the PD-L1 expression in cancer cells. Thirdly, the exact mechanism of CMTM6 regulation in TNBC remains unclear and requires further investigation.

In conclusion, and to the best of our knowledge, this is the first study that has reported a higher expression of CMTM6 in triple-negative compared to HER2- 
A

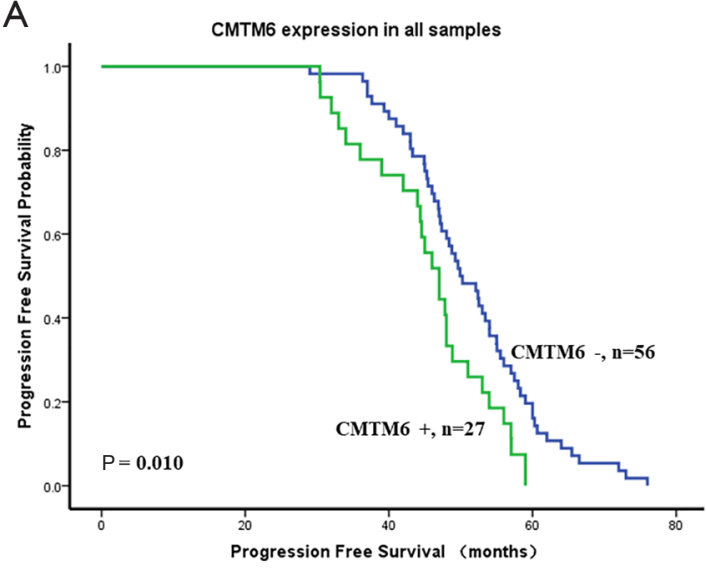

C

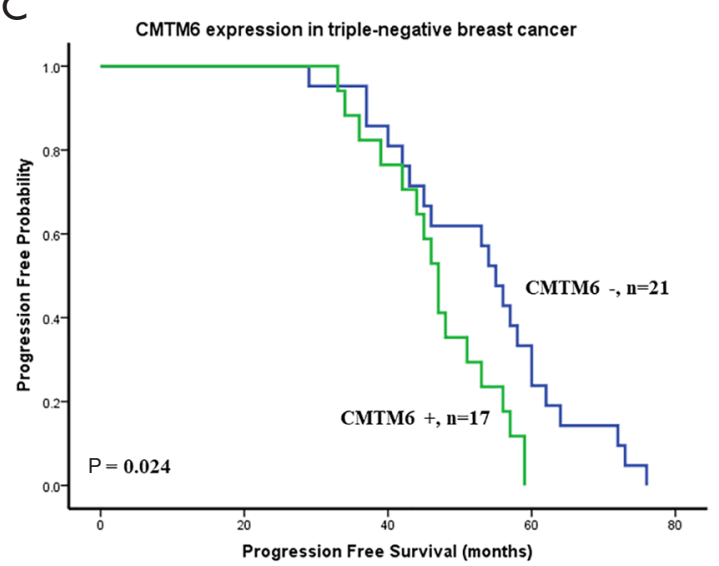

E

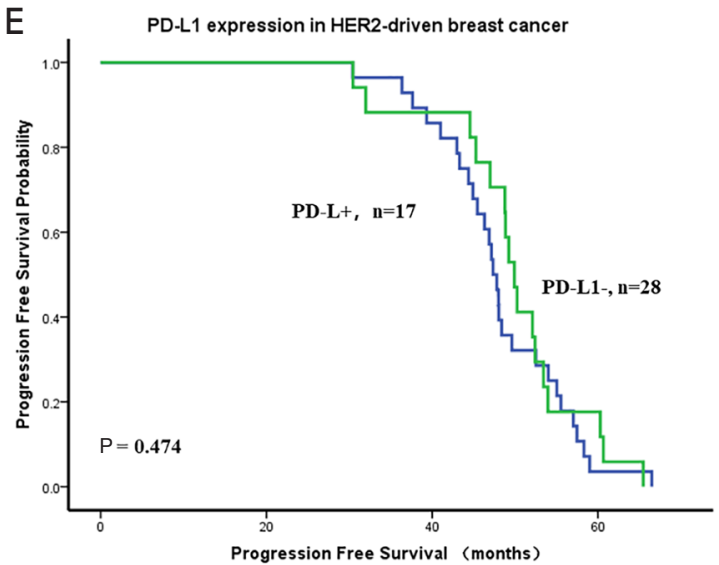

B

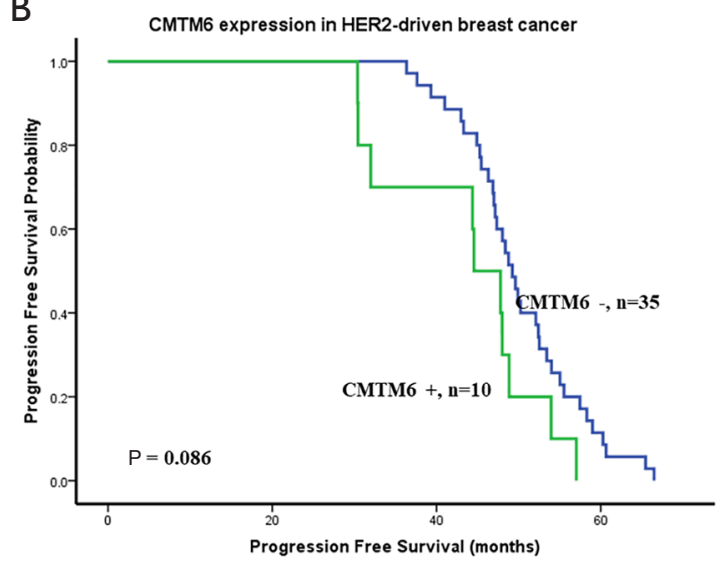

D

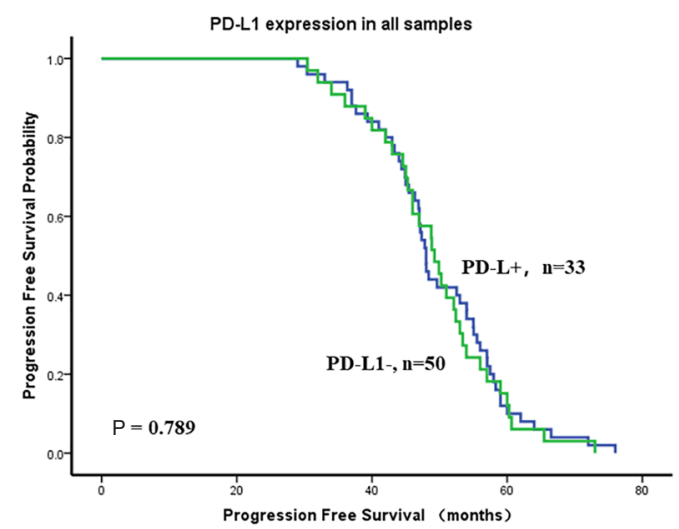

F

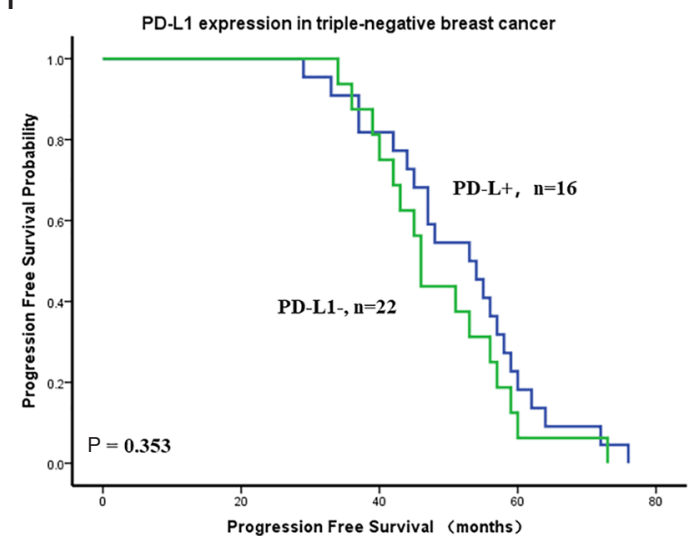

Figure 2 The Kaplan-Meier survival analysis of CMTM6 and PD-L1 expression from 83 primary breast cancers (A,D), 45 primary HER-2 driven breast cancers $(\mathrm{B}, \mathrm{E})$, and 38 primary triple-negative breast cancers $(\mathrm{C}, \mathrm{F})$. $\mathrm{P}$ values for PFS are determined with the log-rank test. N, number of patients. 


\section{Page 10 of 11}

driven breast cancer. The expression of CMTM6 was correlated with PD-L1 in TNBC and could be used as an independent risk factor to predict PFS. The results from this study suggest that CMTM6 may be able to guide prognosis and would become a new potential biomarker of immunotherapies as a supplement to PD-L1 expression in TNBC.

\section{Acknowledgments}

Funding: This study was supported by the Medical Health Science and Technology Project of Zhejiang Provincial Health Commission (2018KY030) and the Natural Science Foundation of Zhejiang Province (LQ21H260001).

\section{Footnote}

Reporting Checklist: The authors have completed the REMARK reporting checklist. Available at http://dx.doi. org/10.21037/atm-20-7616

Data Sharing Statement: Available at http://dx.doi. org/10.21037/atm-20-7616

Conflicts of Interest: All authors have completed the ICMJE uniform disclosure form (available at http://dx.doi. org/10.21037/atm-20-7616). The authors have no conflicts of interest to declare.

Ethical Statement: The authors are accountable for all aspects of the work in ensuring that questions related to the accuracy or integrity of any part of the work are appropriately investigated and resolved. This study was approved by the ethics committee of Zhejiang Cancer Hospital (No. IRB-2020-275). The study was conducted in accordance with the Declaration of Helsinki (as revised in 2013). Because of the retrospective nature of the research, the requirement for informed consent was waived.

Open Access Statement: This is an Open Access article distributed in accordance with the Creative Commons Attribution-NonCommercial-NoDerivs 4.0 International License (CC BY-NC-ND 4.0), which permits the noncommercial replication and distribution of the article with the strict proviso that no changes or edits are made and the original work is properly cited (including links to both the formal publication through the relevant DOI and the license). See: https://creativecommons.org/licenses/by-nc-nd/4.0/.

\section{References}

1. Mittendorf EA, Philips AV, Meric-Bernstam F, et al. PDL1 expression in triple-negative breast cancer. Cancer Immunol Res 2014;2:361-70.

2. Dent R, Trudeau M, Pritchard KI, et al. Triple-negative breast cancer: clinical features and patterns of recurrence. Clin Cancer Res 2007;13:4429-34.

3. Schutz F, Stefanovic S, Mayer L, et al. PD-1/PDL1 Pathway in Breast Cancer. Oncol Res Treat 2017;40:294-7.

4. Topalian SL, Hodi FS, Brahmer JR, et al. Safety, activity, and immune correlates of anti-PD-1 antibody in cancer. $\mathrm{N}$ Engl J Med 2012;366:2443-54.

5. Brahmer JR, Tykodi SS, Chow LQ, et al. Safety and activity of anti-PD-L1 antibody in patients with advanced cancer. N Engl J Med 2012;366:2455-65.

6. Bertucci F, Goncalves A. Immunotherapy in Breast Cancer: the Emerging Role of PD-1 and PD-L1. Curr Oncol Rep 2017;19:64.

7. Zhang M, Sun H, Zhao S, et al. Expression of PD-L1 and prognosis in breast cancer: a meta-analysis. Oncotarget 2017;8:31347-54.

8. Mezzadra R, Sun C, Jae LT, et al. Identification of CMTM6 and CMTM4 as PD-L1 protein regulators. Nature 2017;549:106-10.

9. Gao F, Chen J, Wang J, et al. CMTM6, the newly identified PD-L1 regulator, correlates with PD-L1 expression in lung cancers. Biochem Biophys Rep 2019;20:100690.

10. Burr ML, Sparbier CE, Chan YC, et al. CMTM6 maintains the expression of $\mathrm{PD}-\mathrm{L} 1$ and regulates antitumour immunity. Nature 2017;549:101-5.

11. Vennapusa B, Baker B, Kowanetz M, et al. Development of a PD-L1 Complementary Diagnostic Immunohistochemistry Assay (SP142) for Atezolizumab. Appl Immunohistochem Mol Morphol 2019;27:92-100.

12. Livak KJ, Schmittgen TD. Analysis of relative gene expression data using real-time quantitative PCR and the 2(-Delta Delta C(T)) Method. Methods 2001;25:402-8.

13. Zhang F, Ying L, Jin J, et al. GAP43, a novel metastasis promoter in non-small cell lung cancer. J Transl Med 2018;16:310.

14. Lyons TG. Targeted Therapies for Triple-Negative Breast Cancer. Curr Treat Options Oncol 2019;20:82.

15. Bianchini G, Balko JM, Mayer IA, et al. Triplenegative breast cancer: challenges and opportunities of a heterogeneous disease. Nat Rev Clin Oncol 
2016;13:674-90.

16. Tomioka N, Azuma M, Ikarashi M, et al. The therapeutic candidate for immune checkpoint inhibitors elucidated by the status of tumor-infiltrating lymphocytes (TILs) and programmed death ligand 1 (PD-L1) expression in triple negative breast cancer (TNBC). Breast Cancer 2018;25:34-42.

17. Cimino-Mathews A, Thompson E, Taube JM, et al. PD-L1 (B7-H1) expression and the immune tumor microenvironment in primary and metastatic breast carcinomas. Hum Pathol 2016;47:52-63.

18. Schmid P, Adams S, Rugo HS, et al. Atezolizumab and Nab-Paclitaxel in Advanced Triple-Negative Breast Cancer. N Engl J Med 2018;379:2108-21.

19. Adams S, Schmid P, Rugo HS, et al. Phase 2 study of pembrolizumab (pembro) monotherapy for previously treated metastatic triple-negative breast cancer (mTNBC): KEYNOTE-086 cohort A. J Clin Oncol 2017;35:abstr 1008 .

20. Sharma P, Allison JP. The future of immune checkpoint therapy. Science 2015;348:56-61.

21. Ribas A, Wolchok JD. Cancer immunotherapy using checkpoint blockade. Science 2018;359:1350-5.

Cite this article as: Tian Y, Sun X, Cheng G, Ji E, Yang S, Feng J, Zheng L. The association of CMTM6 expression with prognosis and PD-L1 expression in triple-negative breast cancer. Ann Transl Med 2021;9(2):131. doi: 10.21037/atm-207616
22. Koh YW, Han JH, Haam S, et al. Increased CMTM6 can predict the clinical response to PD-1 inhibitors in non-small cell lung cancer patients. Oncoimmunology 2019;8:e1629261.

23. Nakamura S, Hayashi K, Imaoka Y, et al. Intratumoral heterogeneity of programmed cell death ligand-1 expression is common in lung cancer. PLoS One 2017;12:e0186192.

24. Mamessier E, Birnbaum DJ, Finetti P, et al. CMTM6 stabilizes PD-L1 expression and refines its prognostic value in tumors. Ann Transl Med 2018;6:54.

25. Zhu X, Qi G, Li C, et al. Expression and Clinical Significance of CMTM6 in Hepatocellular Carcinoma. DNA Cell Biol 2019;38:193-7.

26. Guan X, Zhang C, Zhao J, et al. CMTM6 overexpression is associated with molecular and clinical characteristics of malignancy and predicts poor prognosis in gliomas. EBioMedicine 2018;35:233-43.

27. Li X, Chen L, Gu C, et al. CMTM6 significantly relates to PD-L1 and predicts the prognosis of gastric cancer patients. PeerJ 2020;8:e9536.

(English Language Editor: D. Fitzgerald) 


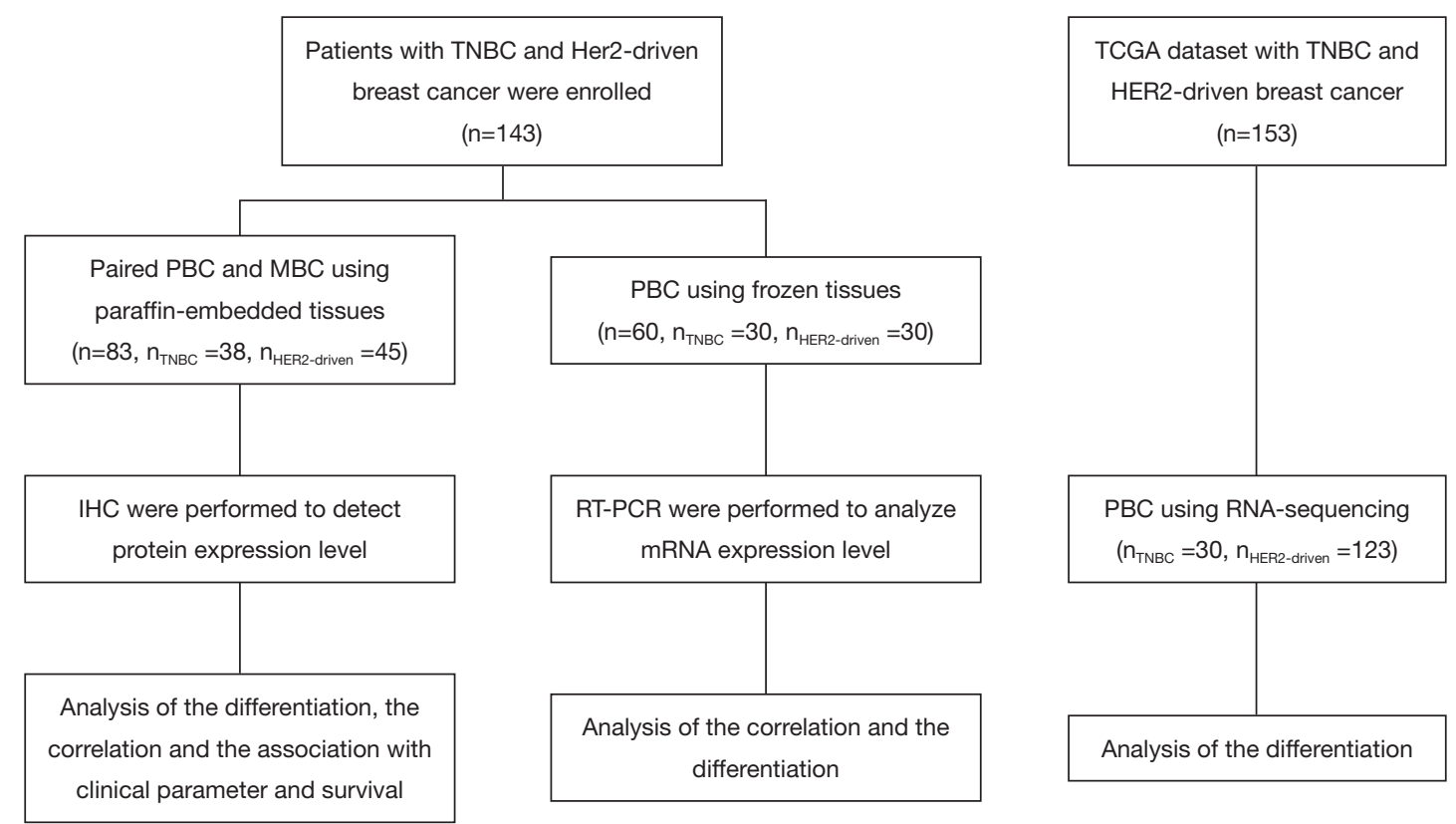

Figure S1 Study scheme. TNBC, triple-negative breast cancer; PBC, primary breast cancer; MBC, metastasis breast cancer; TCGA, The Cancer Genome Atlas.
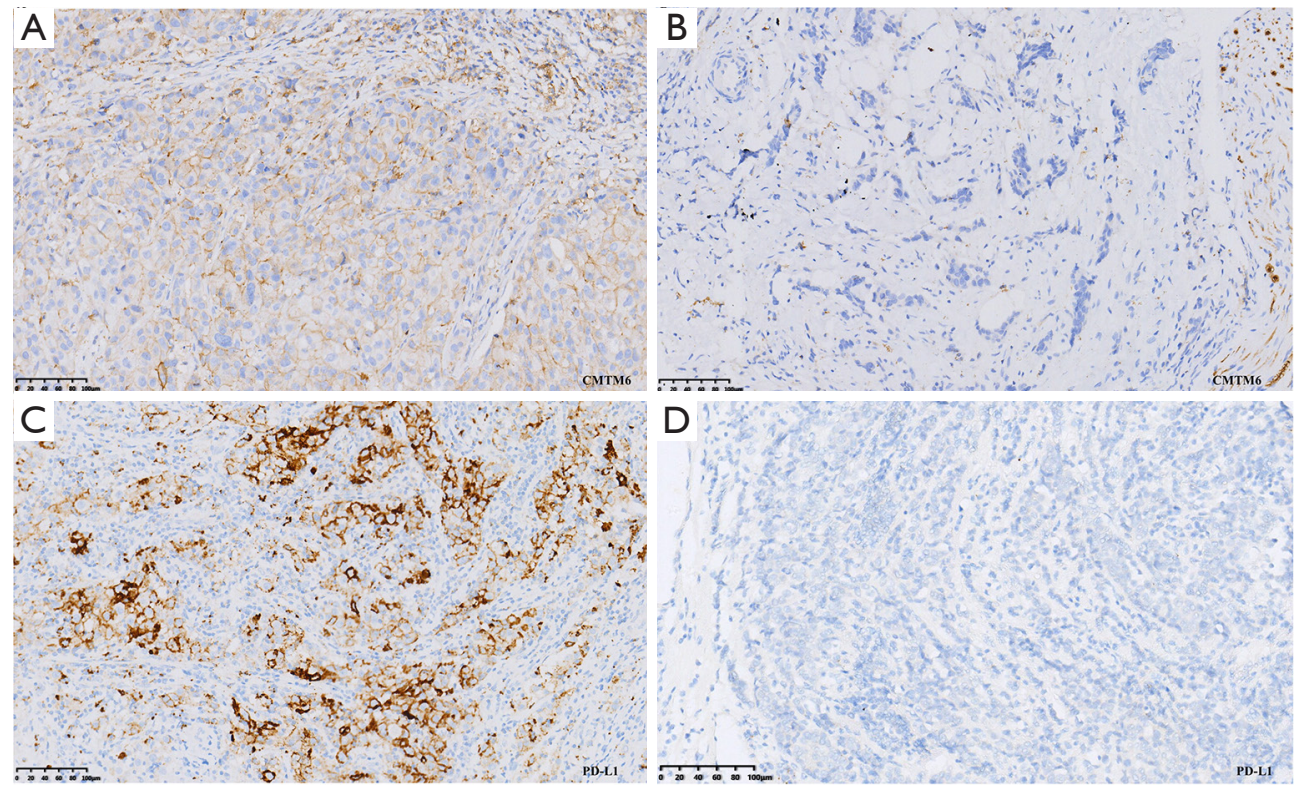

Figure S2 CMTM6 and PD-L1 protein expression in breast cancer using IHC staining. (A) and (C) are positive staining for CMTM6 and PD-L1, respectively. (B) and (D) are negative staining for CMTM6 and PD-L1, respectively. 

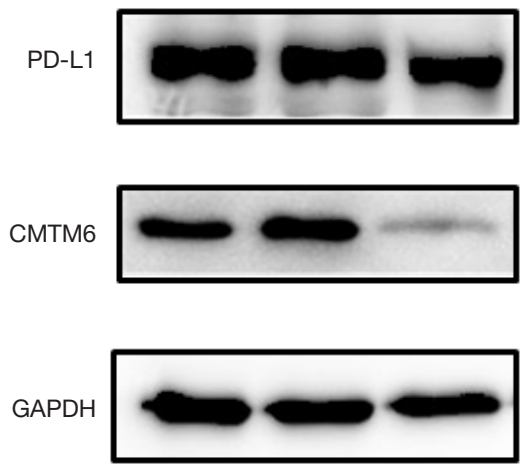

Figure S3 Western blot was used to confirm the detection of IHC.

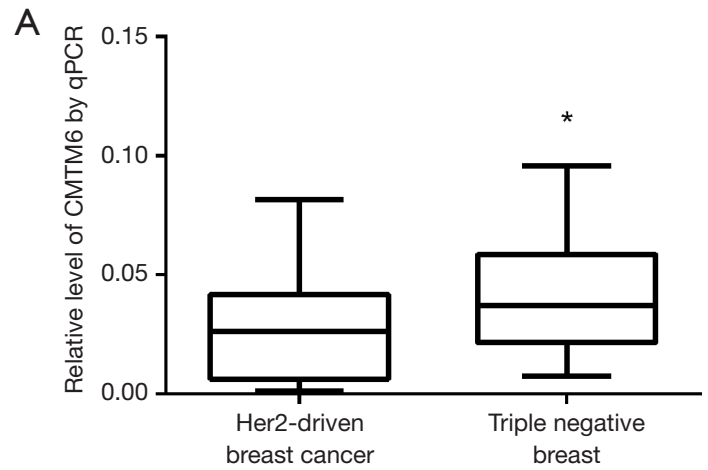

Figure S4 CMTM6 (A) and PD-L1 (B) mRNA expression levels of HER2-driven breast cancer and triple-negative breast cancer. P values are determined based on the Mann-Whitney test. ${ }^{*}, \mathrm{P}<0.05$.

Table S2 CMTM6 and PD-L1 mRNA expression in HER2-driven breast cancer and triple-negative breast cancer expression using TCGA dataset

\begin{tabular}{lccc}
\hline Variable & $\mathrm{N}$ & Mean $\pm \mathrm{SD}$ & $\mathrm{P}$ \\
\hline CMTM6 & & & \\
Her2-driven & 30 & $11.23 \pm 0.626$ & 0.039 \\
Triple negative & 123 & $11.51 \pm 0.728$ & \\
PD-L1 & & & \\
Her2-driven & 30 & $4.7 \pm 1.803$ & 0.283 \\
Triple negative & 123 & $5.05 \pm 1.536$ & \\
\hline
\end{tabular}

CMTM6 and PD-L1 mRNA expression levels of HER2-driven PBC and triple-negative PBC in The Cancer Genome Atlas (TCGA) dataset (see URLs http://cancergenome.nih.gov/). P values are determined based on Student's $t$ test.
Table S1 The mRNA expression level of CMTM6 and PD-L1 in HER2-driven PBC and triple-negative PBC

\begin{tabular}{lccc}
\hline Variable & $\mathrm{N}$ & Median (P25-P75) & $\mathrm{P}$ \\
\hline CMTM6 & & & \\
Her2-driven & 30 & $0.026(0.0062-0.042)$ & 0.036 \\
Triple negative & 30 & $0.037(0.021-0.059)$ & \\
PD-L1 & & & \\
Her2-driven & 30 & $0.00012(0.00005-0.0026)$ & 0.040 \\
Triple negative & 30 & $0.00026(0.00015-0.00043)$ & \\
\hline P values are determined based on Mann-Whitney.
\end{tabular}

B

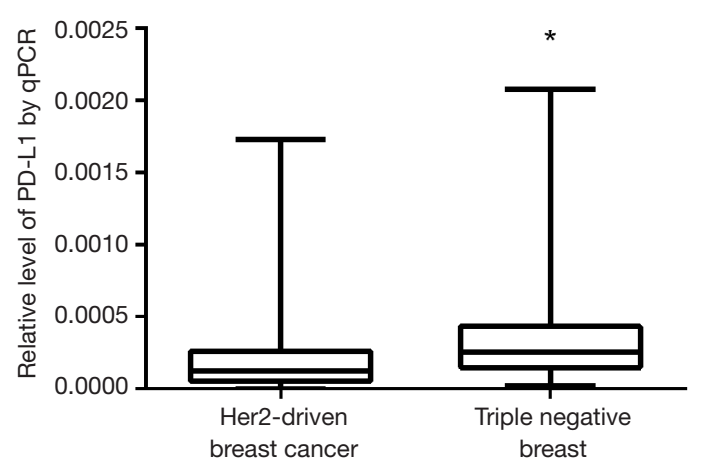

